\title{
Design of a Reference Control Architecture for the Energy Management of Electric Vehicles
}

\section{Dr James Marco (j.marco@cranfield.ac.uk) Prof. Nicholas D Vaughan}

\begin{abstract}
The high voltage network within an electric vehicle (EV) will typically comprise of different energy sources such as fuel cells, batteries and ultracapacitors integrated together through the use of both unidirectional and bidirectional DC-DC converters. Given the multitude of feasible high voltage (HV) network designs, there are obvious advantages in having a unifying control architecture that facilitates the energy management (EM) control task. Within this paper, a control reference architecture (RA) is proposed that can be employed as a template for the design of the energy management control function. The RA exploits the object orientated principles of inheritance and encapsulation in order to achieve the desired non-functional attributes of modularity and control function reuse. Example EM control systems are presented each derived from the same RA, but relating to a different physical configuration of HV network. Simulation results are presented to verify the functional performance of the control systems. In each case, the design trade-offs associated with the functional performance of the EM strategy and the non-functional requirements of modularity and reusability are discussed.
\end{abstract}

\section{Keywords}

Electric Vehicle, Hybrid Electric Vehicle, Control Architecture, Energy Management, SysML, Systems Engineering, Object Orientation

\section{Terms and Abbreviations}

BSFC Best Specific Fuel Consumption

CACSD Computer Aided Control System Design

CCM Context and Causality Model

CPS Continuous Power System 
DC

Direct Current

DM

Decomposition Model

EM

Energy Management

ESC

Energy System Coordinator

EV

Electric Vehicle

HEV

Hybrid Electric Vehicle

HV

High Voltage

IBD

Internal Block Diagram

ICE

Internal Combustion Engine

IGBT

Insulated Gate Bipolar Transistor

IM

Interaction Model

NEDC

New European Drive Cycle

PPS

Peak Power System

RA

Reference Architecture

SD

Sequence Diagram

SM

Strategy Model

SOC

State of Charge

UDDS

Urban Dynamometer Driving Schedule 


\section{Introduction}

The long-term sustainability of electric vehicle (EV) and hybrid electric vehicle (HEV) technology will arguably depend on the ability of the industry to design and integrate such systems in a manner that maximises both the performance and environmental attributes of the new powertrains while concurrently shortening development times and production costs. One means of reducing the development time, costs and therefore the risk, would be the ability to more easily reuse and interchange control functions between the different vehicle concepts.

If the term HEV is used in its most generic sense, in addition to those powertrain topologies that employ an internal combustion engine (ICE), it will also encompass all-electric powertrains that utilise integrated sources of power and energy. Within a hybridised electric powertrain, there is generally one source of steady-state power or energy and one source of peak power. A number of studies have been conducted into electric powertrains in which the steady-state power has been provided by a fuel cell and the peak power by either a battery or an ultracapacitor (Cho et al., 2004; Wenzhong, 2005). Conversely, a number of papers examine concepts in which a high voltage (HV) battery has been employed as the primary source of energy and, once again, an ultracapacitor is used as a transient power buffer (Liqing et al., 2004; Gao et al., 2003; Dixon and Ortuzar, 2002). Based on this definition, it is proposed that a generic hybrid EV will inherently comprise of one or more Continuous Power Systems (CPS) integrated with one or more Peak Power Systems (PPS).

Using these generic terms, Figure 1 presents some example HV network configurations applicable to EVs. Figure 1(a) presents a floating bus option in which the value of voltage on the bus varies with the state of charge $(\mathrm{SOC})$ of the PPS. Figure $1(\mathrm{~b})$ presents the configuration in which a bidirectional converter has been added to the network to stabilise the bus voltage and also to enable the optimisation of the size of the PPS relative to the power and energy requirements of the vehicle. Within the context of this study, the size of the PPS relates to the number of individual cells employed and therefore the operating voltage and energy capacity of the device. Finally, Figure 1(c) represents the HV network in which two energy storage mediums, for example a battery and ultracapacitor have been combined to fulfil the PPS function. The relative merits of each approach are discussed further within (Emadi et al., 2005; Naylor et al., 2006; Marco, 2008), in which consideration is given to:

- the comparative efficiency of each configuration, 
- the relationship between the network topology and the energy storage requirements for the vehicle and,

- the resulting complexity of the energy management (EM) control system.

The research contained within this paper extends that published in (Marco and Vaughan, 2010b) where a modelling approach, based on the Systems Modelling Language (SysML), is proposed that aims to better integrate the traditional computer aided control system design (CACSD) process with the architectural design and documentation methods embodied by (IEEE, 2007). Within (Marco and Vaughan, 2010b), a Control View of the vehicle architecture is formulated. The Control View is comprised of four separate models; the Decomposition Model (DM), the Context and Causality Model (CMM), the Strategy Model (SM) and the Interaction Model (IM). Each of these models exploits and extends the semantics of a SysML Block Definition Diagram (BDD), an Internal Block Diagram (IBD) or a Sequence Diagram. It is beyond the scope of this paper to provide an in depth description of the SysML and the semantics associated with the different diagram types. Further information as to the usability of the language is provided within the SysML specification and other publications and educational texts (OMG, 2008; Hause et al., 2005; Friedenthal et al., 2008). Contained within Appendix $A$ is a summary description of the different models that form the Control View.

Within (Marco and Vaughan, 2010a)(Marco and Vaughan, 2010b), a set of Control View SysML models were developed for a prototype EV that employs a HV network similar to that presented in Figure 1(a). From the improved level of system understanding a Reference Architecture (RA) was proposed that embodied the significant EM control structures and signal flows. Within this paper, the concept of a RA is further extended by first introducing the notion of a base RA. Drawing from the experience of the software domain, the base RA defines the fundamental building block of the architecture that cannot be simplified further (Cloutier and Griego, 2008; Simon Bennett Steve Mcrobb, Ray Farmer, 2006). Once established, the objective is that other EM controller designs can inherent and extend the base RA in order to satisfy their desired functional requirements within the context of their respective physical HV networks. It should be noted that it is not the objective of this study to present a single, generic control architecture for EM applications that can simply be redeployed to any EV concept without modification. The aim of this paper is to present a template for the design of the EM control system for different EV applications. The primary non-functional 
requirement for the control architecture is that it should be modular and as a result have the flexibility to support control integration within different physical domains.

This paper is structured as follows; Section 2 introduces the concept of the RA in relation to both systems design and control engineering. Section 3 introduces the base RA. Consideration is given to the architectural partitioning of the control functionality so as to achieve the desired non-functional attributes of modularity and reusability. Section 4 discusses how the base RA can be extended to support the EM of the HV network presented in Figure 1(b), in which an additional bi-directional converter is added between the HV bus and the PPS. Section 5 discuses how the RA can be further extended to support the EM of the HV network presented in Figure 1(c) in which a battery and ultracapacitor form an integrated PPS. Finally within Section 6, consideration is given as to how an EM strategy for either a series or parallel HEV, employing an ICE, may inherent the proposed EV control architecture as an initial design template.

\section{The Reference Architecture Concept}

A number of publications discuss the application of a RA or design pattern within systems engineering (Cloutier and Griego, 2008; Howard et al., 1999; Grady, 2009; Cloutier and Verma, 2007). It is stated that at a generic level, a RA describes the relationship between a solution and a problem within a defined context. The RA is often expressed at a high level of abstraction in which implementation detail has been omitted and only the key structural and behavioural properties of the proposed solution are presented (Cloutier and Verma, 2007). There are a number of ways in which the use of a RA may support the traditional CACSD activity. For example, a number of publications (IEEE, 2007) advocate the practice of "book shelving" the RA thereby providing an initial design template for future systems and implicitly capturing engineering knowledge and passing that from one programme to another. Secondly, as the implementation of a design matures, models developed using traditional low-level CACSD tools often become much harder to interpret as more "workarounds" are added to the control model. Overtime, the "core" functionality of the desired control system is therefore lost. This in turn, represents a barrier to any attempt to extend, modify or redeploy the control system. Within this context, the RA therefore provides a medium through which design discussions can be undertaken as to how the desired functional and non-functional properties of the control system can be maintained or traded-off against one another. 
There are few examples within the academic literature as to the use of a RA within a control engineering context. One study by (Sanz and Zalewski, 2003) examines the use of control patterns to support the design of a cruise control system. Similarities between pattern-based engineering and objected orientated $(\mathrm{OO})$ design methods are highlighted in which a control pattern is described as an abstract class with the actual implementation being an instantiation of that class.

With specific reference to the EM of EVs and HEVs, studies by (Larson, 2002; Rosario et al., 2006; Beher and Werthschulte, 2009) (Ceraolo et al., 2008; Santos et al., 2006) all address the desire and advantages associated with the adoption of a more modular and reusable control architecture. Within (Larson, 2002) the author describes the creation of a functional architecture for a parallel hybrid powertrain employing a continuously variable transmission (CVT) in which the modularity of the powertrain system controller is maintained by the use of function-wrappers and carefully designed interfaces between the different control elements as one means of reducing subsystem coupling and overall system complexity. The notion of well defined interfaces is extended within (Beher and Werthschulte, 2009) in which an EM structure, based around the Autosar software architecture is presented. Fundamental EM functionality is identified and abstracted away from platform-specific control functions in order to have a strategy that, in principle, is generic and capable of being executed on any Autosar compliant controller. Rather than using a functional decomposition of the control system, a different approach is presented within (Rosario et al., 2006) in which the architecture of the EM controller is layered with respect to the time-domain requirements of the control functions. Three control layers are discussed in which the outer layer represents the steady-state arbitration of power requirements based on the motion of the vehicle. The second layer defines the power split ratio between the two energy storage elements whereas the final layer is concerned with the high-bandwidth feedback control of the power electronics.

The RA concept explored within this paper exploits the principles of object orientation as the primary means of creating of a more modular and reusable control architecture. Generic publications that discuss the adoption of $\mathrm{OO}$ methods as one means of reducing complexity and coupling within the overall design include (Vanderperren et al., 2008; Broy et al., 2006; Fernandes and Lilius, 2004), while ( Broy et al., 2006; Grimm, 2003; Boulanger and Dao, 2008) focus on the design of OO software intensive systems specific to the automotive domain. Of particular interest to this study are the two 
OO properties of encapsulation and inheritance. Encapsulation is broadly defined as the process of hiding the internal structure or functionality of one element from the rest of the system. The motivation being that changes to the encapsulated functionality will not propagate throughout the complete system. Conversely, inheritance deals with the creation or extending of functionality by allowing one element of the architecture to inherit the properties of another.

\section{The Base Reference Architecture}

Contained within this Section is an introduction to the RA and justification for the assertion that it represents the base architecture for the EM of EVs. The existing EM control system, presented in (Marco and Vaughan, 2008) is repartitioned to conform to the requirements of the RA. The performance of the revised control system is presented and compared to the validated performance of the original implementation of the controller. A number of points are highlighted as to the resulting trade-offs between architectural and functional control system design.

\subsection{Design and Justification}

Figure 1(a) presents the HV network for the prototype EV in which the CPS is connected to the HV bus via a DC-DC converter. In parallel to the output stage of the power electronics is the PPS. Discussed in (Marco and Vaughan, 2008) is the detailed design of the control algorithms for the prototype EV in which the CPS is a $26 \mathrm{~kW}$ fuel cell stack and a $17 \mathrm{~F}$ ultracapacitor is employed as the PPS. Since the fuel cell can only supply power to the vehicle, only a unidirectional (boost) converter is required to increase the output voltage from the fuel cell to the $400 \mathrm{~V}$ DC bus. Because the ultracapacitor is directly coupled to the bus, the value of SOC varies with the value of DC voltage.

Figure 2 presents a traditional block diagram control model for the existing design. The control system comprises of an inner current control loop and an outer voltage control loop. The primary objective is to simultaneously regulate the voltage of the ultracapacitor and to limit the rate at which current is drawn from the fuel cell stack. A full explanation of the terms used in the model is provided in (Marco and Vaughan, 2008) and will therefore not be repeated here. The control functionality for the vehicle is hierarchically structured and distributed throughout a number of different Electronic Control Units (ECUs), which are all connected via a controller area network or CAN Bus. The highest level of control authority within the EV is the Vehicle System Controller (VSC). From Figure 2, it can be seen 
that the EM functionality resides, in part, within the VSC and also within the separate ECU, namely the High Voltage Bus Manager (HVBM).

Figures 3(a) and 3(b) present example SysML models of the RA derived from the control implementation of the EM strategy for the prototype EV (Marco and Vaughan, 2010a). Using a SysML IBD, Figure 3(a) presents the main structural properties of the architecture in terms of the interfaces and primary control operations encapsulated within each feature. An example of the complex interactions between the different structural elements of the RA is presented through the use of a SysML sequence diagram as shown in Figure 3(b). It is noteworthy, that Figures $3(a)$ and $3(b)$ do not, in isolation, fully define the concept of the RA. With reference to the proposed Control View, introduced in (Marco and Vaughan, 2010a) and Appendix I, modelling of the RA using the SysML involves the creation of four separate models. Each model defines the decomposition, the structure and behavioural properties of the RA at different levels of abstraction.

From Figure 1(a) and Figure 3(a), it can be seen that the structure of the proposed RA emulates the physical partitioning of the HV network. Within the context of this case study EV, the CPS encapsulates all of the hardware and control functions associated with the fuel cell, whereas the PPS encapsulates all of the hardware and control functions for the ultracapacitor. With respect to the architectural requirements for modularity and extendibility, the following two points should be noted:

- Firstly, the only dependencies that exist between the EM domain and the vehicle motion domain ${ }^{1}$ occur at the coordinator level of the architecture. As reported in a number of studies, including (Beher and Werthschulte, 2009; Browning and Eppinger, 2002), limiting the number of interfaces between different elements of the system, significantly improves its modularity and hence its robustness to subsequent changes. This was found particularly to be the case when the interfaces span multiple levels of system hierarchy.

- Secondly, no direct dependencies are allowed between the CPS and the PPS. This facet of the architecture combined with the encapsulation of control functionality ensures that different technologies can assume the role of the respective CPS and PPS elements.

\footnotetext{
${ }^{1}$ Within the context of the overall control architecture, the vehicle motion domain relates to those control functions concerned with torque allocation and vehicle propulsion.
} 
The control functionality encapsulated within the Energy System Coordinator (ESC), embodies the required vehicle level control functions such as the arbitration of power between the different energy storage mediums and the SOC target setting for the PPS feature. The ESC also acts as the gateway to the rest of the vehicle. This is achieved by transmitting the available power for vehicle acceleration (esc_accel_pow_avail) and regenerative braking (esc_regen_pow_avail) to the vehicle motion domain. Transmitted back to the ESC from the vehicle motion domain is a measure of vehicle speed (vmc_veh_spd). Since, within this case study an ultracapacitor is employed as the PPS, the management of SOC is inherently a function of vehicle speed ${ }^{2}$. A demand or target SOC (esc_soc_dmd) is transmitted from the ESC to the PPS feature. Based on the SOC demand and an internal measure of energy, the power request field (pps_pow_req) is calculated. This signal defines the power required by the PPS feature to maintain the SOC to the target value. The CPS receives a power demand from the ESC (esc_pow_dmd). The CPS feature sends back a measure of available power that can support vehicle propulsion.

The authors propose that this EM control architecture for a hybrid EV constitutes the base RA since further decomposition or simplification of the system is not possible, for the following reasons:

- Firstly, without the PPS the CPS must act as a load-follower in that it must meet the full load requirements of the driver. As a result, there is no requirement for a vehicle-level EM strategy.

- Secondly, if the DC-DC converter was removed from the HV network, there would be no direct means of control integration. Partitioning of the load power between the PPS and the CPS would be purely passive and based on the inherent dynamic properties of the two physical subsystems.

- Finally, since the CPS is only able to supply power to the vehicle, only a unidirectional DC-DC converter is required for subsystem integration as opposed to a bidirectional (buck-boost) converter if the CPS was able to support energy storage.

\footnotetext{
${ }^{2}$ It is noteworthy that if different technology options are to be employed then further considerations, such as cell temperature and cell discharge rates may also need to be taken into account. However, such additional control functions would tend to be encapsulated within the localised management of the control feature and not used as interfaces at the architectural level of the control system.
} 


\subsection{Performance Simulation}

The existing control algorithms, presented in (Marco and Vaughan, 2008), were re-engineered to conform to the partitioning and interface requirements of the RA shown in Figure 3(a). The revised control system has been implemented within Simulink and integrated with the previously validated EV powertrain model (Marco and Vaughan, 2008).

Figure 4 presents the simulation results obtained when the model has been exercised over the UDDS legislative drive cycle. The figure shows the vehicle speed profile, the SOC of the ultracapacitor in terms of both the demand (esc_soc_dmd) and the measured value and finally the power flows on the DC bus. From the results presented, it can be seen that the performance of the system is comparable to those published within (Marco and Vaughan, 2008).

The vehicle range predicted from the original implementation of the control system and the simulation of the EM strategy based on the new RA are presented in Table 1. As it can be seen, for three different legislative drive cycles, similar results are obtained between the two versions of the control system, thereby verifying their functional equivalence. As discussed in the following sub-section, the differences between the two sets of results can, in part, be explained by considering the interaction that exists between the functional design of the control system and its architectural partitioning.

\subsection{Design Discussion}

An observation made during the verification of the revised system is that the feedback algorithm employed to control the rate and magnitude of the power drawn from the fuel cell, must be recalibrated when the actuation signal within the control loop was changed from a current request to a power request. With the latter, a new variable gain representing the DC bus voltage is included within the closed loop dynamics, therefore changing the set-point response of the algorithm. This conclusion is verified by means of examining the frequency response for the linearised system. This example highlights the interdependency that may exist between the functional performance of the strategy and the architectural partitioning of the control system. This conclusion has obvious implications for the introduction and re-use of legacy control functions within vehicle systems while trying to maintain common interfaces, as discussed in (Rosario et al., 2006; Beher and Werthschulte, 2009). 
With respect to the PPS, the two outputs defining the acceleration and regenerative braking power available (pps_accel_pow_avail, pps_regen_pow_avail) present one example of encapsulation and, as discussed within (Broy et al., 2006; Fernandes and Lilius, 2004; Grimm, 2003), the frequent design trade-off that occurs between increased localised design effort and architectural robustness. Both measures are an internal function of SOC within the PPS. From the localised design perspective of that feature transmitting SOC would imply the simplest solution to implement. However, this implies that the ESC feature would have to transform that SOC value into a corresponding power value in order to perform the required vehicle-level arbitration. Such a computation would require the ESC to know the internal attributes of the PPS and as a result, an inherent dependency or coupling would exist between the two. For example, if the size of the ultracapacitor or battery was to change, then modifications to the control architecture would propagate beyond the PPS and thereby reduce the overall modularity of the design.

\section{Extending the Base Reference Architecture for a Fixed Bus Network}

The aim of this Section is to discuss how the base RA, presented in Section 3, can be extended to accommodate the EM function within a physical HV network that employs an additional bidirectional DC-DC converter as shown in Figure 1(b). The purpose of the additional power electronics is to stabilise the bus voltage and also to provide greater management of the power that flows into and out of the PPS. Particular consideration is given to the architectural design of the strategy rather than the detailed design of the feedback control algorithms. However, sufficient detail is presented to enable the reader to assess the general approach taken to the EM task with further information being provided within (Marco, 2008). For reference, a schematic of the EM control structure is presented in Figure B.1 (Appendix B).

\subsection{System Overview}

For the purpose of this case study, the HV network presented in Figure 1(b) is employed. Figure 5 presents an example CCM for the system that highlights the causality within the physical domain and the primary control relationships within the electrical architecture. As highlighted within Appendix $A$, it is noteworthy that further models exist that define the Control View for this system, each defining either the decomposition of the system, the system structure or its behaviour at a different level of abstraction. 
The case study application is comprised of a fuel cell, the output voltage of which is stepped-up by a DC-DC boost converter to the vehicle bus. As before, an ultracapacitor is employed to buffer the fuel cell from the transient power demands associated with vehicle acceleration and also to act as an energy storage medium, thereby enabling regenerative braking to occur. However on this instance, an additional bi-directional (buck-boost) converter is employed between the ultracapacitor and the DC bus. Since the bidirectional converter introduces two additional power electronic switches $\left(u_{1}\right.$ for boost operation and $\mathrm{u}_{2}$ for buck operation), further degrees of freedom are added to the control system (Marco, 2008; Maksimovic et al., 2001).

With respect to Figure 5 and Appendix $B$, the fuel cell is controlled via a single feedback loop around the stack and the boost converter. The design objective being to manage the magnitude and rate at which power is drawn from the fuel cell. These control objectives are realised through a low-pass filter in series with a saturation as part of the pre-filtering strategy for the set-point to the control loop. The set-point power demand applied to the fuel cell control loop is comprised of two separate components. The first is an estimate of the vehicle power demand for a given value of driver input, whereas the second relates to the power required by the ultracapacitor to maintain its SOC to the target value. When the ultracapacitor contains excess energy, the difference between the actual and demand SOC is negative resulting in less power being drawn from the fuel cell for a given driver demand. Conversely, if the SOC of the ultracapacitor is lower than the target value, additional power is drawn from the fuel cell to both meet the vehicle load requirements and also to recharge the device.

The value of DC bus voltage is controlled via a voltage control loop and two inner current control loops. One current loop is required for each of the switching devices within the bidirectional converter. For the case in which the bus voltage is too low, $u_{1}$ is regulated (with $u_{2}$ held off) to allow additional power to be sourced from the ultracapacitor to raise the bus voltage to the desired level. Conversely, during a regenerative braking event, for example, in which the bus voltage may increase above the target value the duty-cycle applied to $u_{2}$ is increased (with $u_{1}$ held off) so that additional energy can flow from the DC link to the ultracapacitor.

From the description above, it can be seen that this control strategy represents a load averaging, charge sustaining approach to the EM of a hybrid EV. The control system is comprised of fived nested 
control loops. In comparison to the control schematic presented in Figure 2, the detail of the control problem is therefore considerably different to that associated with the base RA.

\subsection{Architectural Partitioning of the EM Strategy}

Figure 6 presents how the RA for the EM task within an hybrid EV employing a fixed bus electrical network can employ the same structure as the base RA while extending its functionality in the following two ways:

- Firstly, an additional interface is required between the ESC and the vehicle motion domain. The measure of vehicle power required (veh_pow_dmd) is used by the ESC to generate a power demand to the CPS feature. With respect to the case study vehicle discussed in Section 4.1 this value is primarily used to derive the set-point to the fuel cell control loop.

- The second modification to the base RA relates to changes in the encapsulated functionality within both the PPS and the ESC features. Additional control functions within the PPS are required to manage the bidirectional converter, whereas within the ESC additional control functions are required to derive the set-point to the fuel cell (cps_pow_dmd).

From the complete Control View, Figure 7 presents an example DM that shows how the control functionary and physical subsystems within the complete electrical architecture can be encapsulated within the three RA elements (the ESC, the CPS and the PPS).

\subsection{Performance Verification}

Figure 8 presents the realisation of the control system within a Simulink model. Extending the EV powertrain model employed in Section 3.2 to include the representative efficiency and dynamics of the bidirectional converter, Figure 9 presents the simulated performance of the EM control system as the vehicle is exercised over the same UDDS drive cycle. The Figure focuses on the new functionality of the revised portion of the control system, namely the active management of the energy level within the ultracapacitor. The Figure presents the SOC within the device (both demand and actual), the flow of current into and out of the device and the control signals applied to $u_{1}$ and $u_{2}$. It should be noted that positive current represents the ultracapacitor being charged and therefore, as shown in Figure 9, $\mathrm{u}_{1}$ is held open-circuit and the control system regulates the energy flow by varying the duty-cycle 
applied to $u_{2}$. The inverse relationship is true when the power electronics are operating as a boost converter.

Given the limitation of this case study, in that it does not directly relate to an existing EV, it is not possible to experimentally validate the results presented. However, the performance of the EM strategy is consistent with the notion of a charge sustaining, load averaging control approach, as discussed within (Cho et al., 2004; Schupbach and Balda, 2003; Cegnar et al., 2004). The resultant range of the vehicle over the UDDS drive cycle was found to be $522 \mathrm{~km}$, which is comparable to that discussed in Section 3 given the differences in the physical properties of the vehicle and the calibration of the control algorithms.

\subsection{Design Discussion}

The extensions to the base RA described in Section 4.2 relate to the particular design of the EM strategy discussed in Section 4.1. Further modifications to the RA may be desirable given a specific vehicle application. For example, within the ESC feature, the powertrain efficiency model used to estimate the power demand to the fuel cell has been implemented as a calibration. A refinement to the control system may include the use of model-based techniques, in which a real-time model of the EV powertrain is used to provide a more accurate estimate of the desired power draw from the CPS. Such a decision may necessitate additional interfaces between the ESC and rest of the control architecture within the vehicle.

With respect to the PPS class, the required value of bus voltage is defined internally as a calibration, since it relates primarily to the localised management (or encapsulation) of PPS resources. Another design option would be for the target value of HV bus voltage to be transmitted by the ESC. Such a design decision would be logical if the value of bus voltage was correlated to additional vehicle-level performance attributes. For example, it may be desirable to lower the bus voltage under regenerative braking conditions thereby allowing the vehicle to capture more energy as the vehicle decelerates through lower speeds.

For both design options discussed above, the enhanced functional performance of the control system would come at the cost of increased coupling within the control architecture. As a result, this may in turn reduce the modularity and reusability of the overall design. As stated in the Introduction, it is not 
the objective of this study to define a generic EM strategy suitable for all possible EV concepts, but rather to present a RA that allows reasoned engineering decisions to be made as to the complexity and interconnectivity of the complete system in relation to the desired functional design of the control algorithms.

\section{Extending the Base Reference Architecture for a Hybrid PPS Feature}

The final case study, relates to the modifications required to the base RA to support the EM of an electrical architecture in which, as shown in Figure 1(c), a hybrid PPS is employed within the vehicle. A number of recent studies have reviewed the control strategies required to integrate a HV battery with an ultracapacitor (Gao et al., 2003; Ozatay et al., 2004). Often this has been done to hybridise a fuel cell within an EV, in which the battery and ultracapacitor combination are employed to buffer the fuel cell from transient demands, to act as a range-extender for the EV and to provide a source of energy for the fuel cell balance-of-plant (Wenzhong, 2005; Emadi et al., 2005).

\subsection{System Overview}

Figure 10 presents a CCM showing the causality within the physical domain of the HV electrical network and the primary control functions. As within Section 4.1, the aim of this section is to provide a qualitative description of the control algorithms required for systems integration. A detailed discussion into the design of the feedback and supervisory algorithms is beyond the scope of this paper. For reference, a schematic of the EM control structure is presented in Figure B.2 (Appendix B).

As with the previous example, the fuel cell is controlled via a single feedback control loop around the fuel cell stack and the boost converter. The set-point power demand to the control loop is a filtered estimate of the power demand placed on the HV bus by the electrical machines. Since the fuel cell is only able to support vehicle acceleration and cruise, the set-point demand is always positive.

Under vehicle acceleration, because the power supplied by the fuel cell is less than the demand value, the ultracapacitor that is directly coupled to the HV bus will discharge and the SOC of the ultracapacitor will reduce. As the value of SOC falls below the desired value, additional power is drawn from the HV battery to maintain the SOC of the ultracapacitor. The EM strategy during regenerative braking aims to first maintain the SOC of the ultracapacitor at the desired value. 
If the value of ultracapacitor SOC exceeds the desired value, then the bidirectional converter, acting as a buck converter, is controlled to draw power from the HV bus and use the excess power to recharge the HV battery as well as the ultracapacitor. Irrespective of whether the bidirectional converter is employed in a buck or boost mode of operation, control of the power electronic switches ensures that the battery C-rating is not exceeded.

\subsection{Architectural Partitioning of the EM Strategy}

For the system introduced above, Figure 11 presents an example DM that shows how the control functionary and physical subsystems within the complete electrical architecture can be encapsulated within the three RA elements (the ESC, the CPS and the PPS). The novel use of recursion is employed within the control architecture as one means of reducing the complexity of the design. Recursion within the architecture of a system relates to the reuse of common elements or patterns, often at different levels of system hierarchy (Grady, 2009; Cloutier and Verma, 2007; Yavuz and Bradshaw, 2002). Studies have highlighted that because the same element is reused the modularity and understandability of the design are improved (Cloutier and Verma, 2007; Yavuz and Bradshaw, 2002).

Figure 12 presents the revised PPS feature for the RA that contains the recursive architectural design. Within the context of the EM functionality introduced in Section 5.1, the following modifications are required to the base RA to facilitate the use of a hybrid PPS within the electrical architecture of an EV:

- Because the PPS.CPS ${ }^{3}$ feature now encapsulates the HV battery, which can both sink and source energy, the PPS.CPS control element must contain an additional operation to calculate the regenerative power that can be stored in the battery. An additional interface between the PPS.CPS and PPS.ESC features is also required to integrate this functionality with the overall EM strategy.

\footnotetext{
${ }^{3}$ The notation; PPS.CPS is used to describe the hierarchy within the architecture, namely the CPS feature contained within the PPS element.
} 
- As described in Section 3, the top-level ESC feature calculates the SOC demand for the PPS (esc_soc_dmd). The PPS.ESC does not need to recalculate this value again and as a result it will contain some redundant functionality and also an unused interface (vmc_veh_spd).

\subsection{Performance Verification}

Figure 13 shows the simulated performance of the RA when the control strategy has been implemented within the Mathworks toolset. The figure presents the performance of the EM algorithms as the vehicle traverses the UDDS drive cycle. The Figure presents the load power and the power contribution from the fuel cell, the ultracapacitor and the HV battery. In accordance with the qualitative description of the EM strategy discussed in Section 5.1, during vehicle acceleration the ultracapacitor meets the majority of the driver demand, while power from the fuel cell is ramped-up at a slower rate to prevent oxygen starvation within the stack. Also at a much slower rate of discharge, additional energy is also drawn from the battery. During the regenerative braking event, the majority of the power on the HV bus is stored within the ultracapacitor, with the excess energy also being used to recharge the HV battery. The control of the bidirectional converter, which is encapsulated within the PPS.CPS feature, ensures that the battery C-rating is not exceeded. It is noteworthy that due to the relatively modest vehicle acceleration and deceleration rates associated with the drive cycle the amount of power that is both sunk and sourced from the battery is less than that found during more aggressive periods of driver demand.

\subsection{Design Discussion}

It should be noted that other approaches to the EM task, to that discussed above, are possible and can be supported by the RA. Two such examples are described below for reference:

The first enhancement to the functionality of the system would be to enable the fuel cell to replenish the battery SOC should the latter reduce below a threshold value. To enable this enhancement, an additional power request output for the PPS.CPS feature is required. This output, in addition to the existing pps_pow_req signal at the higher level of the EM hierarchy could be utilised to demand excess power from the fuel cell. The benefit of the additional functionality would therefore come at the cost of an additional interface; from a low-level of the architecture to the higher level control functions. 
The second modification, relates to the different strategic approach to the EM problem. From the discussion presented in Section 4.1, it can be seen that the load essentially acts as an external disturbance to the HV bus. The SOC of the ultracapacitor is increased or decreased and when required to keep the SOC of the ultracapacitor close to the target value, additional energy is sourced or sunk from the HV battery at a rate and magnitude appropriate for that specific technology. Another approach would be for the top-level ESC feature to arbitrate the power distribution between the fuel cell and the HV battery. Within such a scheme a local cost-function optimisation strategy may be employed within the ESC. Any surplus or deficit of power on the HV bus would charge/discharge the ultracapacitor. In order to facilitate this strategy, an additional esc_pow_dmd interface would be required between the top-level ESC feature and the PPS feature which would then be propagated through to the low-level PPS.CPS feature that encapsulates the HV battery.

As with the previous case studies introduced in this paper, the two design options presented above relate to possible trade-offs that exist between increased control system functionality and the architectural modularity of the design. They further highlight the use of the RA as a convenient medium through which reasoned engineering decisions can be made as to the interconnectivity of the complete system in relation to the desired functional design of the control system.

\section{Discussion}

The simulation and analysis presented within the proceeding sections, have focussed on the extension of the base RA to support the EM control function within three EV concepts that employ different HV electrical networks. Contained within this section is a brief discussion as to how the base RA may be employed as a template to support the design of the EM control system within a HEV employing an ICE.

Within a series hybrid vehicle, irrespective of the control methodology employed, the generic aim is often to use the HV battery to suppress the transient demands placed on the ICE. As a result, since the ICE is mechanically decoupled from the road-wheels it is able to operate within its most efficient region. A typical approach is to allow the ICE to operate around the Best Specific Fuel Consumption (BSFC) region, while maintaining the SOC of the battery within a defined upper and lower limit. With reference to the base RA presented in Figures 3(a) and 3(b), it can be seen that the CPS class would 
intuitively encapsulate all of the control functions for the combined engine and generator. Conversely, the PPS would encapsulate the localised management of the HV battery.

The ESC feature would compute the amount of power required from the ICE to recharge the battery (esc_pow_dmd). This value of power, combined with an internal measure of engine speed, would allow the CPS to calculate the required value of torque to load the engine. The ESC would define the target SOC for the battery (esc_soc_dmd) to ensure that there is always sufficient energy to assist in vehicle acceleration and available capacity to store energy via regenerative braking.

The CPS would transmit the available power from the ICE (cps_pow_avail) taking into account any operating strategies that may limit the available power from the ICE. An extension to the base RA may be the inclusion of a desired power interface between the CPS and ESC classes. This interface would enable the ICE to define the desired power to be drawn thereby allowing it to maximise its localised efficiency. In terms of the PPS, it would transmit the power available (pps_accel_pow_avail, pps_regen_pow_avail), which within this context would define the availability of power for the vehicle motion domain. The power request interface (pps_pow_req) between the PPS and the ESC features enables the PPS to request additional power from the ICE to replenish the battery SOC.

With the interfaces defined above, the control engineer is free to design the internal control algorithms for each feature while maintaining the modularity and transferability of the overall design. Conversely an existing PPS class may be employed within the series hybrid control scheme, thereby allowing the manufacturer to redeploy the same control functions between different EV and HEV concept vehicles.

Extending the base RA to support a parallel hybrid application is however more challenging. The primary reason for this is that unlike within an EV and a series HEV in which the allocation of power and torque can be done independently, within a parallel hybrid vehicle the management of torque within the powertrain implicitly defines the required arbitration of power between the ICE and the different electrical subsystems. The scope to which a parallel hybrid EM strategy can inherent and extend the base RA, taking into account the feasible powertrain configurations, control methodologies, and vehicle hybridisation ratios will be the subject of a future research publication. As part of this future study, consideration will be given to the required design compromises associated 
with the architectural requirements for modularity and transferability and the ability of the EM algorithm to meet its functional objectives of good vehicle driveability and low fuel consumption.

\section{Conclusions}

If the term HEV is used in its most generic sense, in addition to those powertrain topologies that employ an ICE, it will also encompass all-electric powertrains that utilise hybridised sources of power and energy, such as fuel cells, batteries and ultracapacitors. A combination of bidirectional or unidirectional power electronic subsystems will typically be employed to integrate such subsystems to form the vehicle's HV network. Given the design options in terms of the different technology subsystems and possible network topologies, there are obvious advantages in having a unifying control architecture that facilitates the EM control task. Within this paper, the concept of a control RA is proposed and its use verified by considering its ability to support HV network options that use different power electronic and energy storage technologies. The ability of the control architecture to support extensions in the physical domain represents a realistic scenario in which the automotive manufacturer may wish to redeploy the same control functions onto different EV or HEV platforms. Simulation results have been presented that verify the functional performance of the control systems. In addition, for each of the case study vehicles, example design trade-offs are discussed that highlight the use of the RA concept in supporting the control system design process and in particular the challenging comprise between the feature content of the control system and the modularity and transferability of the control architecture.

\section{Acknowledgements}

To be completed after peer review

\section{References}

Beher, U. and Werthschulte, K. (2009), "Energy Management as Configurable System Software Function", SAE Technical Paper Series: 2009-01-0516, .

Boulanger, J. -. and Dao, V. Q. (2008), "Requirements engineering in a model-based methodology for embedded automotive software", 2008 IEEE International Conference on Research, Innovation and Vision for the Future in Computing and Communication Technologies, Ho Chi Minh City, China, pp. 263-268. 
Browning, T. R. and Eppinger, S. D. (2002), "Modeling Impacts of Process Architecture on Cost and Schedule Risk in Product Development", IEEE Transactions on Engineering Management, vol. 49, no. 4, pp. 428-442.

Broy, M., Pretschner, A., Salzmann, C. and Stauner, T. (2006), "Software-Intensive Systems in the Automotive Domain: Challenges for Research and Education", SAE Technical Paper Series, vol. 2006-01-1458.

Cegnar, E. J., Hess, H. L. and Johnson, B. K. (2004), "A purely ultracapacitor energy storage system hybrid electric vehicles utilizing a based DC-DC boost converter", Hess, H. L. (ed.), in: 19th IEEE Applied Power Electronics Conference and Exposition, APEC'04, Vol. 2, 22nd-26th February, Anaheim, California, USA, pp. 1160.

Ceraolo, M., di Donato, A. and Franceschi, G. (2008), "A General Approach to Energy Optimization of Hybrid Electric Vehicles", IEEE Transactions on Vehicular Technology, vol. 57, no. 3, pp. 1433-1441.

Cho, H. Y., Cho, H. Y., Gao, W. and Ginn, H. L. (2004), "A new power control strategy for hybrid fuel cell vehicles A new power control strategy for hybrid fuel cell vehicles", Gao, W. (ed.), in: Power Electronics in Transportation, 2004, pp. 159.

Cloutier, R. and Griego, R. (2008), "Applying object oriented systems engineering to complex systems", 2008 IEEE International Systems Conference Proceedings, pp. 515-520.

Cloutier, R. J. and Verma, D. (2007), "Applying the Concept of Patterns to Systems Architecture", Systems Engineering, vol. 10, no. 2, pp. 138-154.

Dixon, J. W. and Ortuzar, M. E. (2002), "Ultracapacitors + DC-DC converters in regenerative braking system", IEEE Aerospace and Electronic Systems Magazine, vol. 17, no. 8, pp. 16-21.

Emadi, A., Rajashekara, K., Williamson, S. S. and Lukic, S. M. (2005), "Topological overview of hybrid electric and fuel cell vehicular power system architectures and configurations", IEEE Transactions on Vehicular Technology, vol. 54, no. 3, pp. 763-770.

Fernandes, J. M. and Lilius, J. (2004), "Functional and Object-Oriented Views in Embedded Software Modeling", Proceedings of the 11th IEEE International Conference and Workshop on the Engineering of Computer-Based Systems, 24 - 27 May, Brno, Czech Republic, pp. 378 - 387.

Friedenthal, S., Moore, A. and Steiner, R. (2008), A Practcal Guide to the SysML: The Systems Modelling Language, 1st Edition ed, Elsevier.

Gao, L., Dougal, R. A. and Liu, S. (2003), "Active power sharing in hybrid battery/capacitor power sources", Dougal, R. A. (ed.), in: 18th IEEE Applied Power Electronics Conference and Exposition, APEC '03, Vol. 1, 9th-13th February, Florida, USA, pp. 497-503.

Grady, J. O. (2009), "Universal Architecture Description Framework", Systems Engineering, vol. 12, no. 2, pp. 91-116.

Grimm, K. (2003), "Software technology in an automotive company - major challenges", 25th IEEE International Conference on Software Engineering, Portland, Oregon, USA, pp. 498-503. 
Hause, M., Thom, F. and Moore, A. (2005), "Inside SysML", IEE Electronics Systems and Software, vol. 3, no. 3, pp. 20-25.

Howard, A., Kochhar, K. and Dilworth, J. (1999), "Application of a Generic Manufacturing Planning and Control System Reference Architecture to Different Manufacturing Environments", Proceedings of the Institution of Mechanical Engineers, Part B: Journal of Engineering Manufacture, vol. 213, no. 4, pp. 381-391.

IEEE (2007), "Systems and Software Engineering - Recommended Practice for Architectural Description of Software-Intensive Systems", ISO/IEC 42010 IEEE Std 1471-2000 First edition 200707-15, .

Larson, M. (2002), "A Reusable System Architecture for Hybrid Powertrains", SAE Technical Paper Series, , no. 2002-01-2808.

Liqing, S., Wei, C., Leiyan, Y., Lianghui, Y., L, Y. and S, F. (2004), "Design and Analysis of Pure Electric Bus Jointly Powered by Lithium-Ion Battery and Ultra Capacitor", SAE Technical Paper Series, vol. 2004-01-0368.

Maksimovic, D., Stankovic, A. M., Thottuvelil, V. J. and Verghese, G. C. (2001), "Modeling and simulation of power electronic converters", Proceedings of the IEEE Transactions, vol. 89, no. 6, pp. 898-912.

Marco, J. and Vaughan, N. D. (2010a), "Architectural Modelling of an Energy Management Control System Using the SysML", International Journal of Vehicle Design (accepted for publication), vol. -, no. -, pp. ---.

Marco, J. and Vaughan, N. D. (2010b), "Integration of Architectural Modeling Using the SysML within the Traditional Automotive CACSD Process", Proceedings of the International Conference on Control (UKACC) 7th - 10th September, Coventry University, UK, .

Marco, J. (2008), "Electrical Architectures for Hybrid Vehicles: Implications for Modelling and Control", Proceedings of the 2008 International Conference on Control (UKACC), 2008, 2nd - 4th September, University of Manchester, UK, .

Marco, J. and Vaughan, N. D. (2008), "The Control-Oriented Design and Simulation of a High Voltage Bus Management Strategy for use within Hybrid Electric Vehicles", International Journal of Vehicle Systems Modelling and Testing, vol. 2, no. 4, pp. 345-368.

Naylor, S. M., Pickert, V. and Atkinson, D. J. (2006), "Fuel Cell Drive Train Topologies - Computer Analysis of Potential Systems", Power Electronics, Machines and Drives, 2006. The 3rd IET International Conference on, pp. 398-403.

OMG (2008), The Systems Modelling Language, v1.1, http://www.omgsysml.org/, .

Ozatay, E., Zile, B., Anstrom, J. and Brennan, S. (2004), "Power distribution control coordinating ultracapacitors and batteries for electric vehicles", Zile, B. (ed.), in: Proceedings of the 2004 American Control Conference, Vol. 5, Boston, USA, pp. 4716-4721. 
Rosario, L., Luk, P. C. K., Economou, J. T. and White, B. A. A. (2006), "A Modular Power and Energy Management Structure for Dual-Energy Source Electric Vehicles", Luk, P. C. K. (ed.), in: IEEE Vehicle Power and Propulsion Conference, VPPC '06. Windsor, UK, pp. 1.

Santos, F., Trovao, J., Marques, A., Pedreiras, P., Ferreira, J., Almeida, L. and Santos, M. (2006), "A Modular Control Architecture for a Small Electric Vehicle", IEEE Conference on Emerging Technologies and Factory Automation, pp. 139-144.

Sanz, R. and Zalewski, J. (2003), "Pattern-Based Control Systems Engineering", IEEE Control Systems Magazine, vol. 23; 23, no. 3, pp. 43-60-60.

Schupbach, R. M. and Balda, J. C. (2003), "The role of ultracapacitors in an energy storage unit for vehicle power management", Balda, J. C. (ed.), in: 58th IEEE Vehicular Technology Conference VTC 2003-Fall, Vol. 5, 6th-9th October, Orlando, Florida USA, pp. 3236-3240.

Simon Bennett Steve Mcrobb, Ray Farmer (2006), Object-Oriented Systems Analysis and Design Using UML, 3rd ed, McGraw-Hill Higher Education, UK.

Vanderperren, Y., Mueller, W. and Dehaene, W. (2008), "UML for Electronic Systems Design: A Comprehensive Overview", Design Automation for Embedded Systems, vol. 12, no. 4, pp. 261-292.

Wenzhong, G. (2005), "Performance comparison of a fuel cell-battery hybrid powertrain and a fuel cell-ultracapacitor hybrid powertrain", IEEE Transactions on Vehicular Technology, vol. 54, no. 3, pp. 846-855.

Yavuz, H. and Bradshaw, A. (2002), "A new conceptual approach to the design of hybrid control architecture for autonomous mobile robots", Journal of Intelligent and Robotic Systems: Theory and Applications, vol. 34, no. 1, pp. 1-26. 
10 Appendix A

\begin{tabular}{|l|c|l|}
\hline \multicolumn{1}{|c|}{ Model Name } & $\begin{array}{c}\text { SysML } \\
\text { Diagrams } \\
\text { Used }\end{array}$ & Description of Use \\
\hline $\begin{array}{l}\text { Decomposition } \\
\text { Model (DM) }\end{array}$ & BDD & $\begin{array}{l}\text { The highest level of abstraction within the Control View, in } \\
\text { that both the structure and the behaviour of the system have } \\
\text { been omitted. The main function of this model is to clearly } \\
\text { show the principle system elements and their respective } \\
\text { hierarchy within the system boundary. }\end{array}$ \\
\hline $\begin{array}{l}\text { Context and } \\
\text { Causality Model }\end{array}$ & IBD & $\begin{array}{l}\text { The purpose of the context and causality model is twofold; } \\
\text { firstly it defines the system boundary. Secondly, it defines the } \\
\text { causality within the physical domain. }\end{array}$ \\
\hline $\begin{array}{l}\text { Strategy Model } \\
\text { (SM) }\end{array}$ & IBD & $\begin{array}{l}\text { The strategy model shows the functional integration of the } \\
\text { control system and the dependencies that exist between the } \\
\text { different control features, independent of their final } \\
\text { deployment to specific hardware platforms. }\end{array}$ \\
\hline $\begin{array}{l}\text { Interaction Model } \\
\text { (IM) }\end{array}$ & SD & $\begin{array}{l}\text { The interaction model addresses the behaviour of the system } \\
\text { and highlights the complex interactions between the different } \\
\text { elements of the control system at a feature level, irrespective } \\
\text { of the physical deployment of the control functions to different } \\
\text { ECUs within the network. }\end{array}$ \\
\hline
\end{tabular}

Table A.1: Summary of the different Control View models (Marco and Vaughan, 2010b) 


\section{Appendix B}

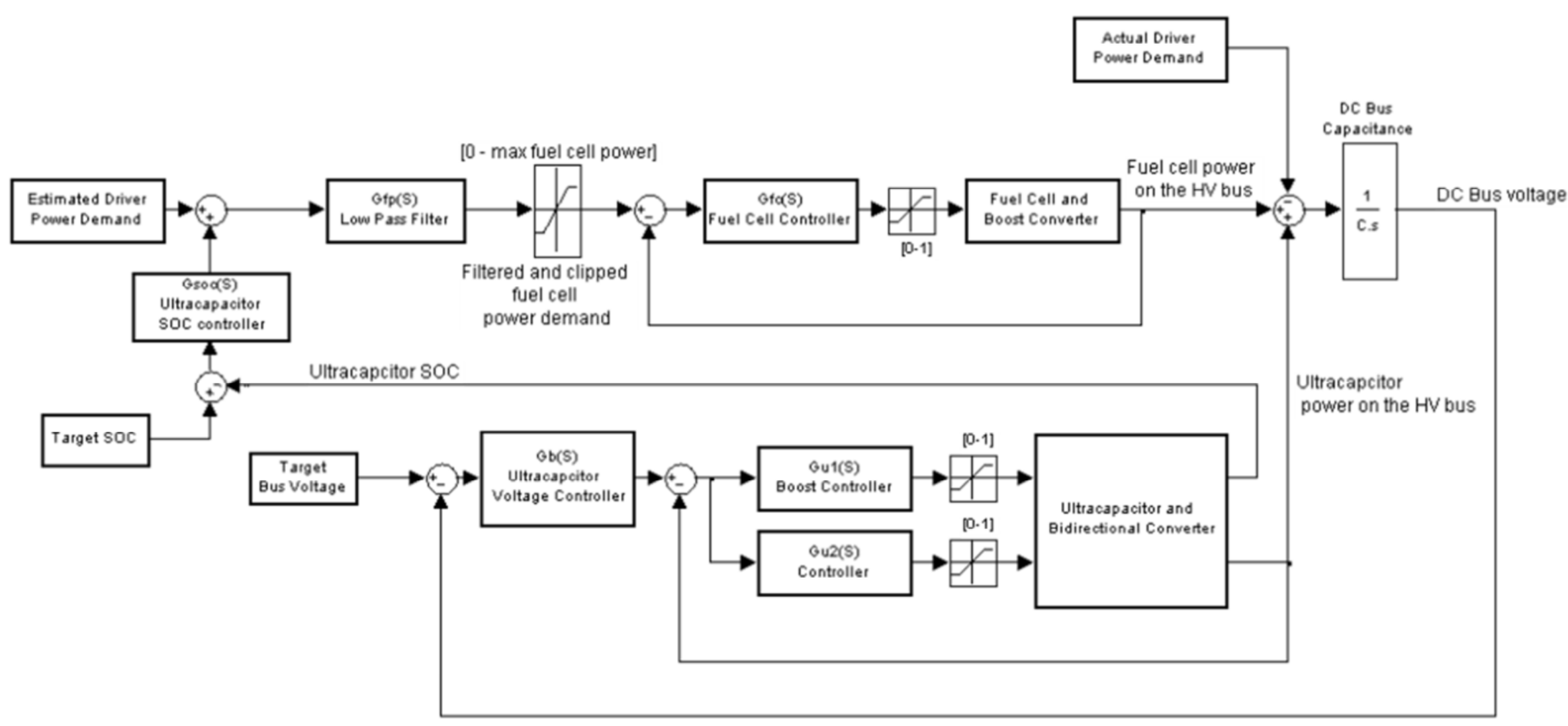

Figure B.1: Schematic for the EM Ccontrol structure for the electrical architecture employing a bidirectional DC-DC converter 


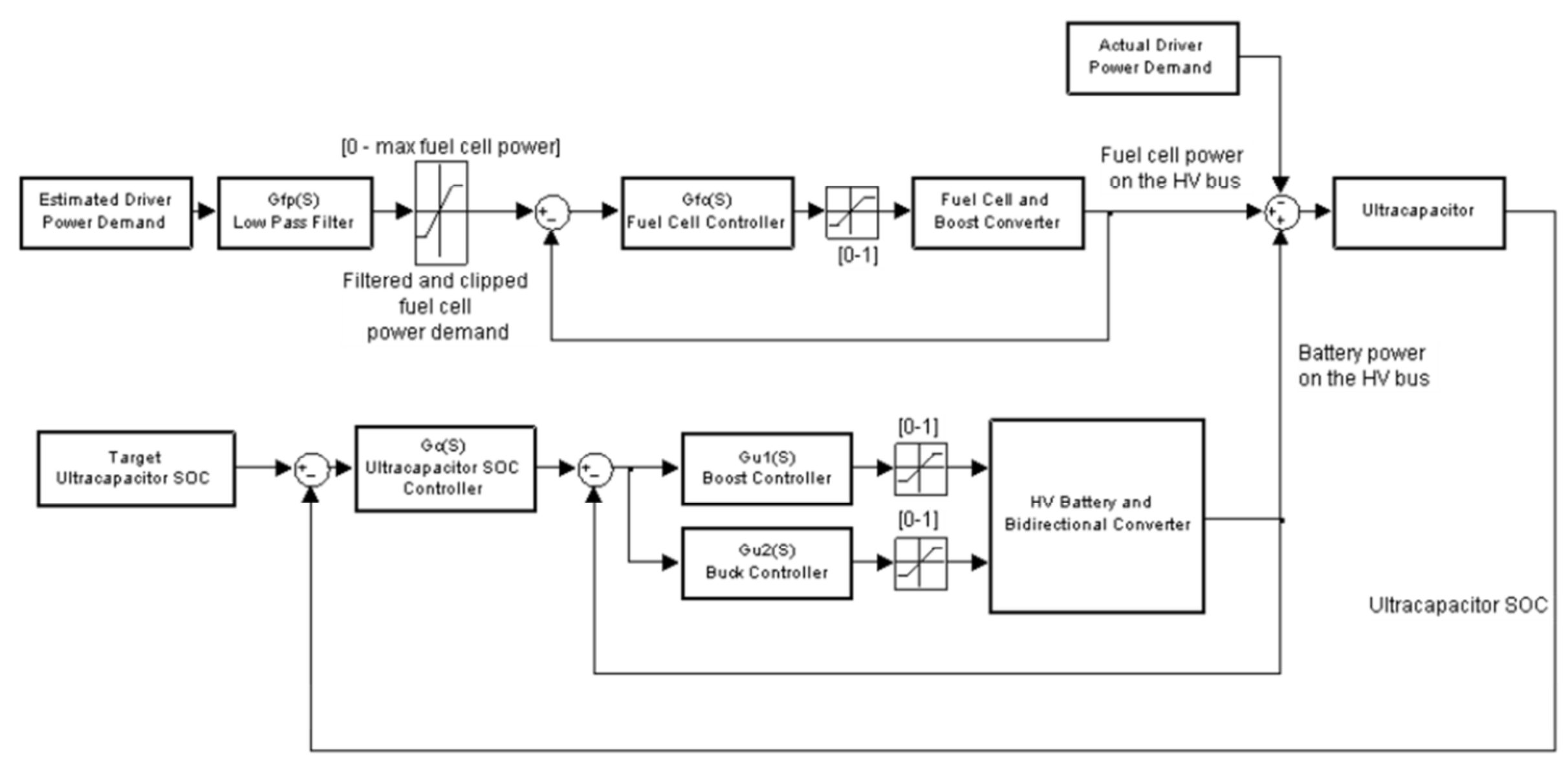

Figure B.2: Schematic for the EM control structure for the electrical architecture employing a hybrid PPS (HV battery and ultracapacitor) 


\section{Tables}

\begin{tabular}{|c|c|c|}
\hline $\begin{array}{c}\text { Vehicle Range } \\
\text { (Original } \\
\begin{array}{c}\text { Controller } \\
\text { Implementation) }\end{array}\end{array}$ & $\begin{array}{c}\text { Vehicle Range } \\
\text { (Revised } \\
\text { Controller based } \\
\text { on the RA) }\end{array}$ & Variation \\
\hline \multicolumn{3}{|c|}{ NEDC } \\
\hline $455 \mathrm{~km}$ & $472 \mathrm{~km}$ & $3.7 \%$ \\
\hline \multicolumn{3}{|c|}{ UDDS } \\
\hline $486 \mathrm{~km}$ & $501 \mathrm{~km}$ & $3.1 \%$ \\
\hline \multicolumn{3}{|c|}{ US06 } \\
\hline $295 \mathrm{~km}$ & $311 \mathrm{~km}$ & $5.4 \%$ \\
\hline
\end{tabular}

Table 1: A comparison of EM performance metrics over different legislative drive cycles 


\section{Figures}
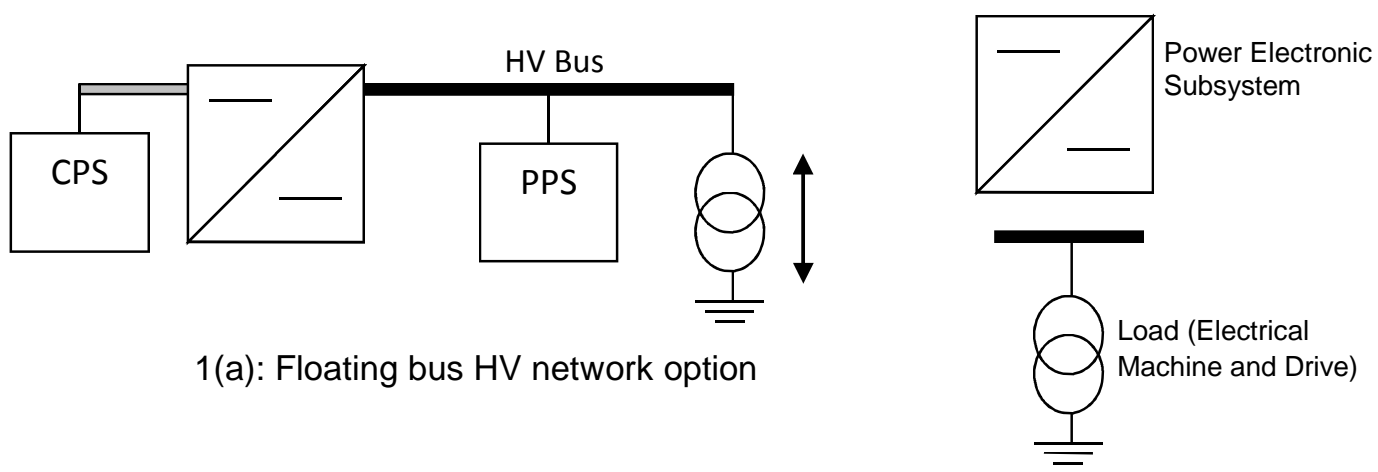

1(a): Floating bus HV network option

Load (Electrical

$\stackrel{I}{=}$

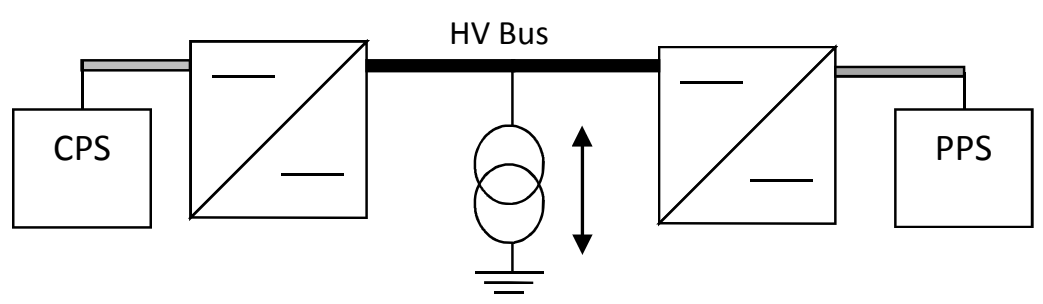

1(b): Fixed bus HV network option

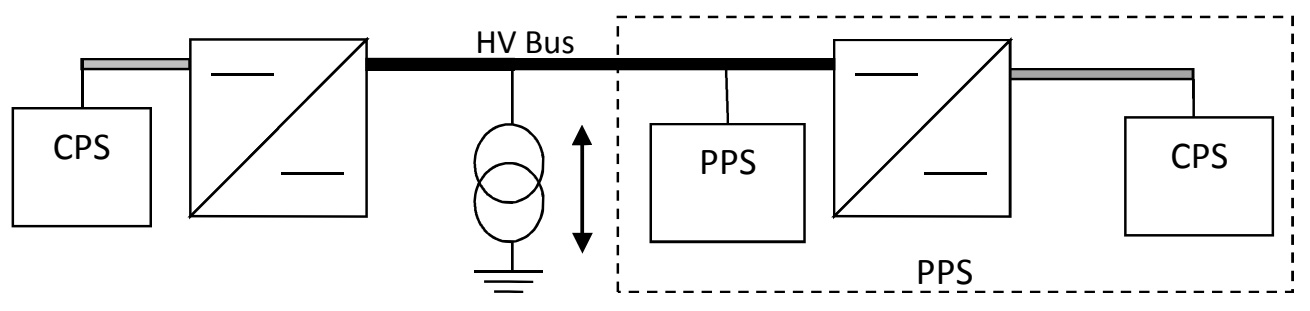

1(c): Fixed bus HV network option with a hybrid PPS subsystem

Figure 1: Example HV Network options for a Hybrid EV showing the Integration of power electronics with the continuous and peak power systems 


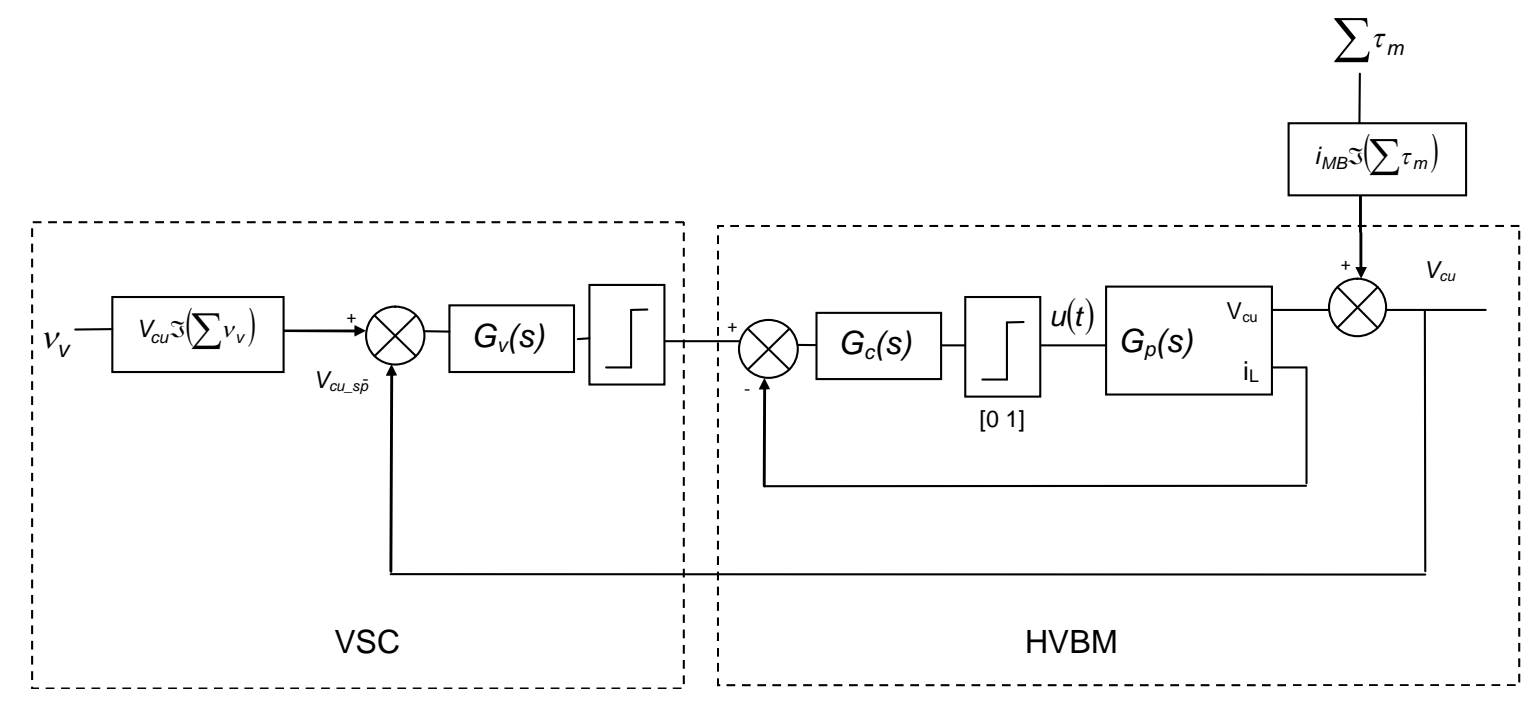

Figure 2: Structure of the EM control system for the prototype EV 


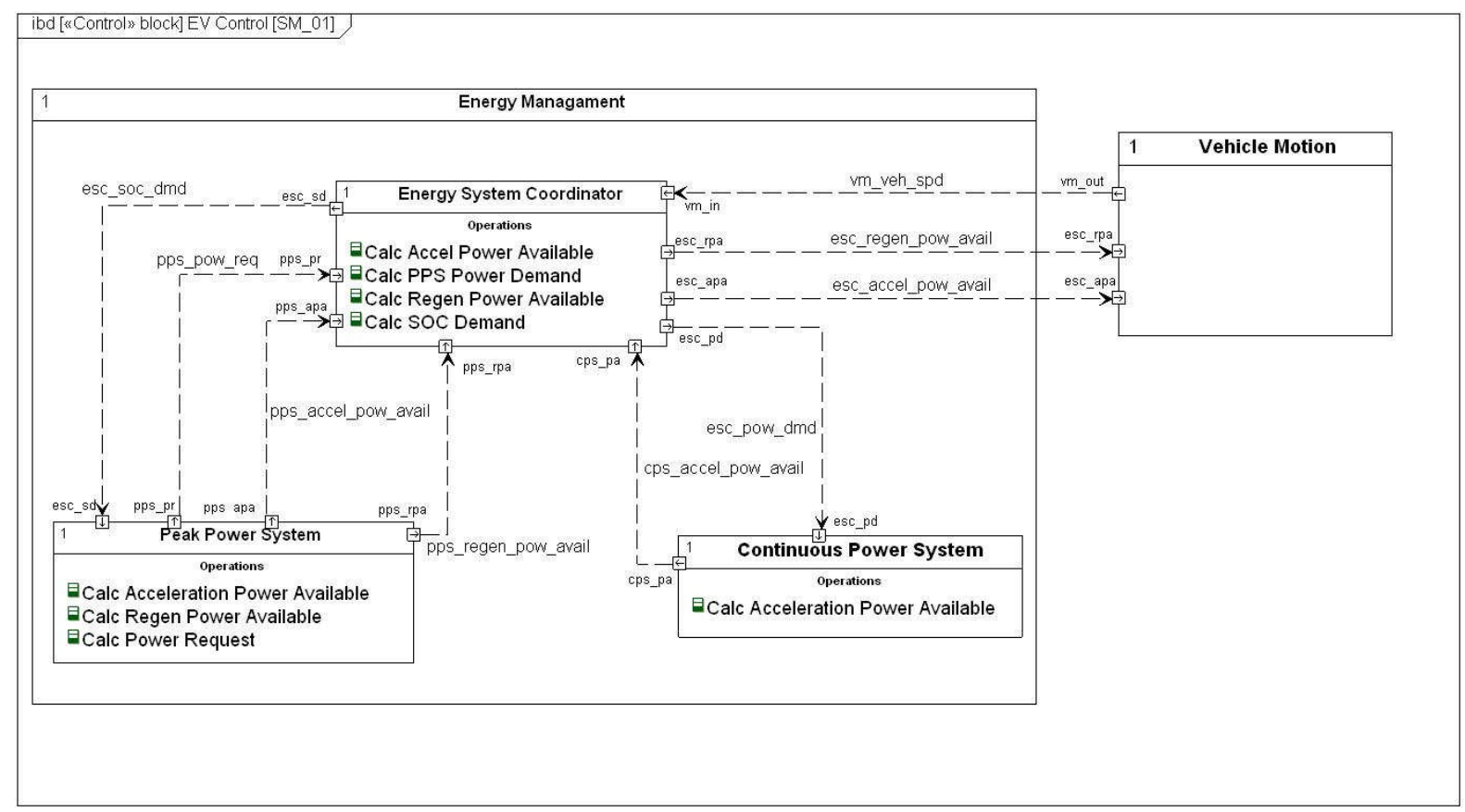

Figure 3(a): SysML IBD showing the internal structure of the base RA for the EM control system

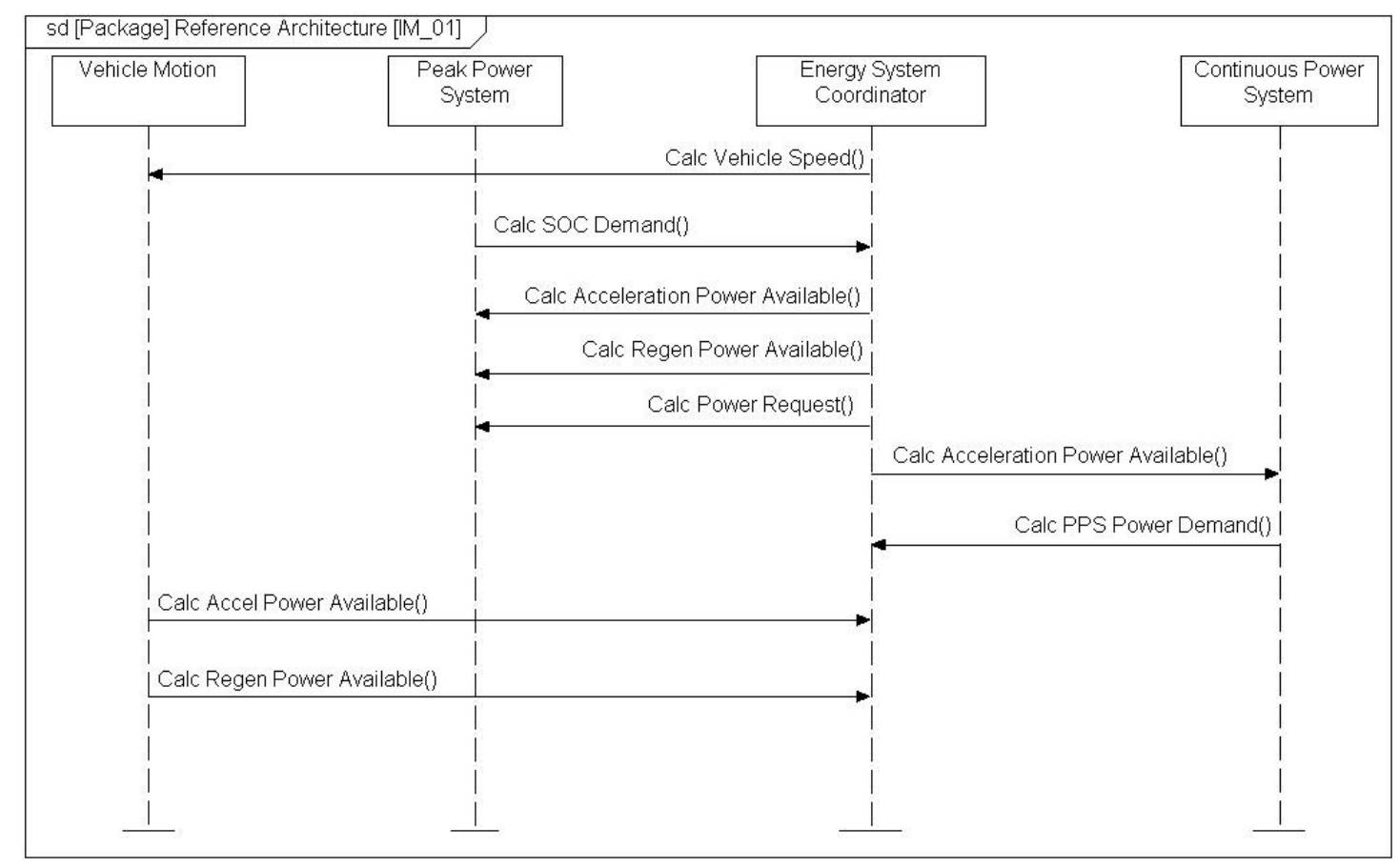

Figure 3(b): SysML sequence diagram showing example controller behaviour of the Base RA for the EM control system 

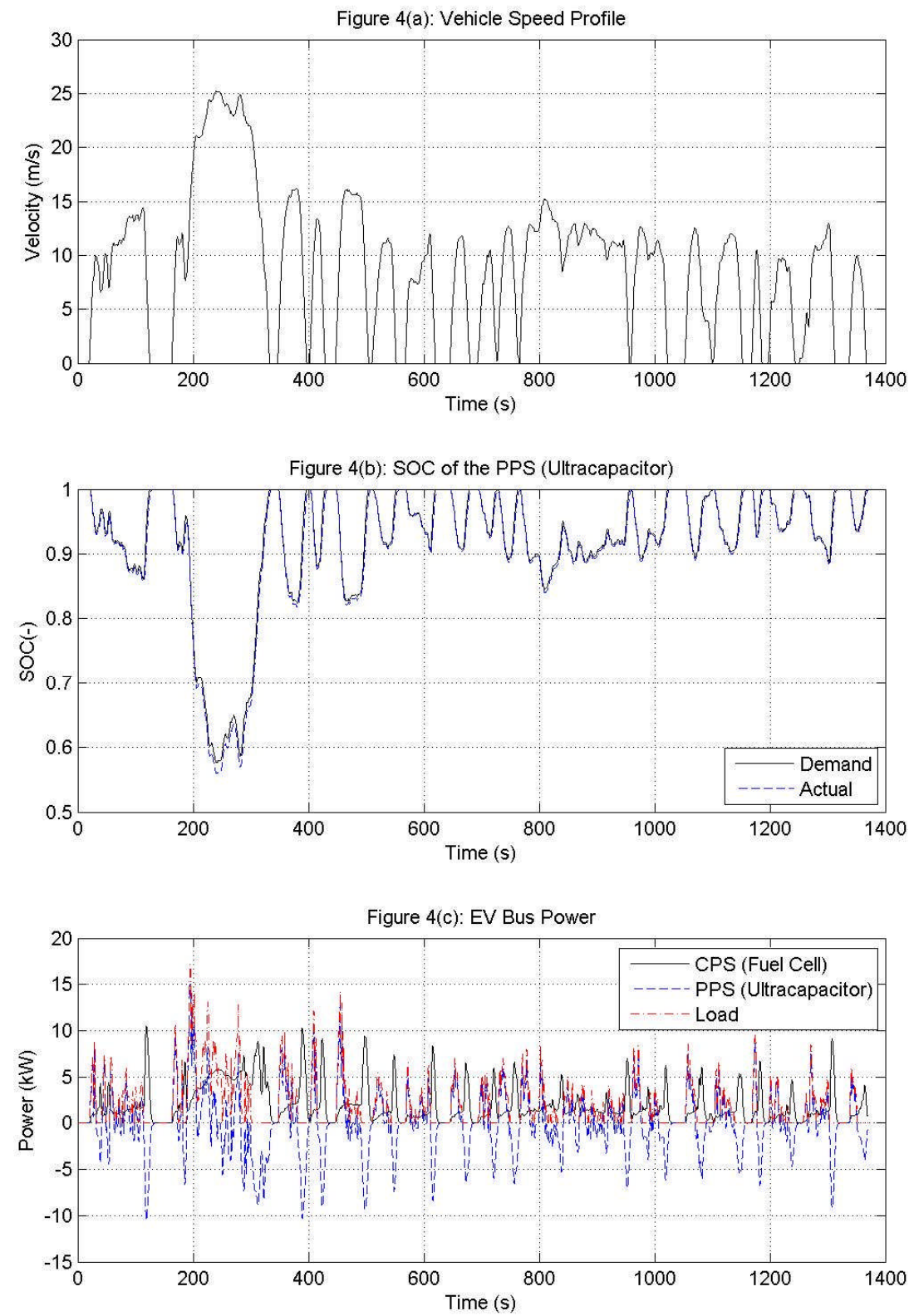

Figure 4: Simulations results of the base reference architecture over the UDDS drive cycle 


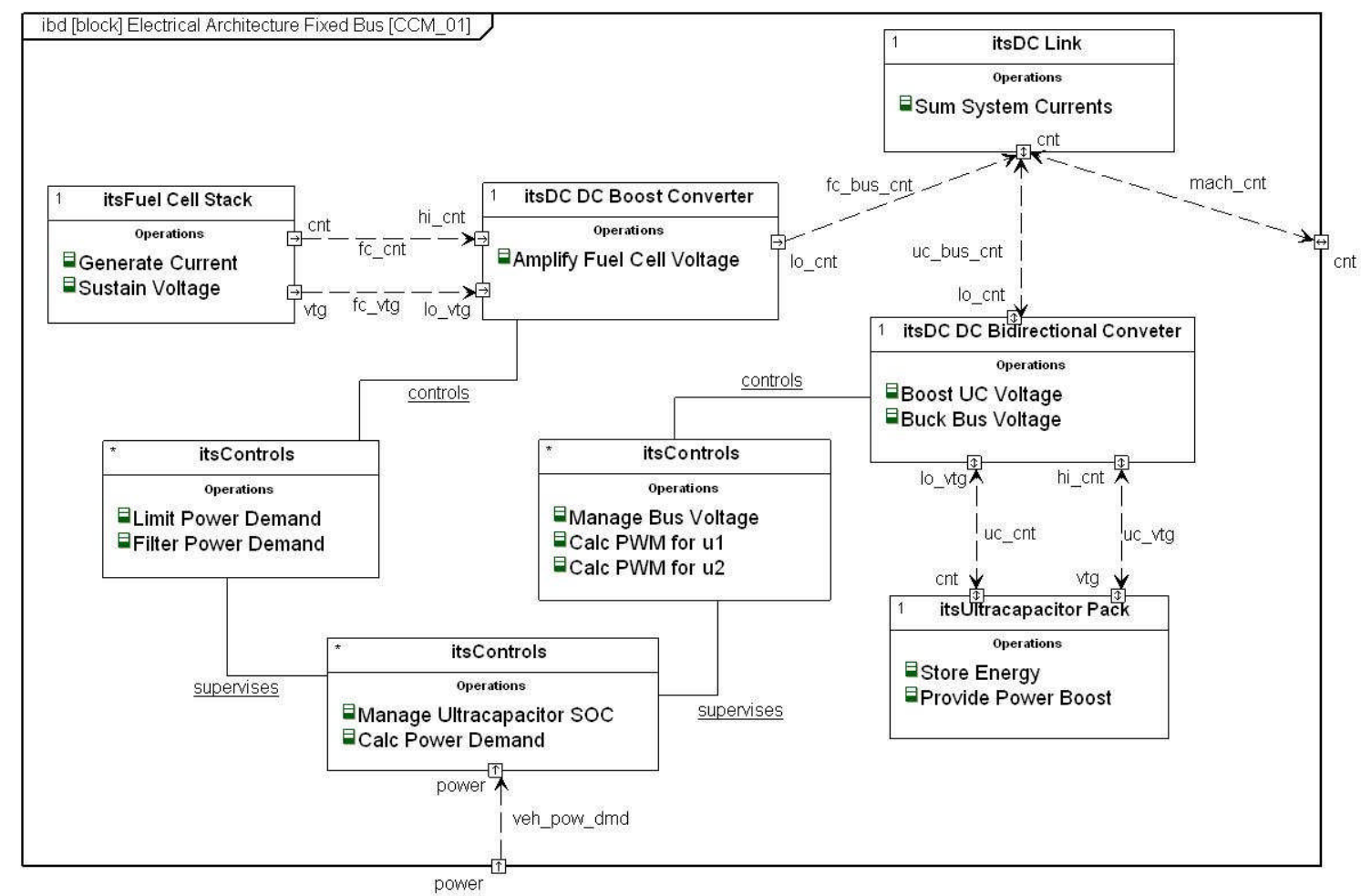

Figure 5: Context and causality model for the revised EV electrical architecture employing an additional bidirectional converter between the PPS and DC bus. 


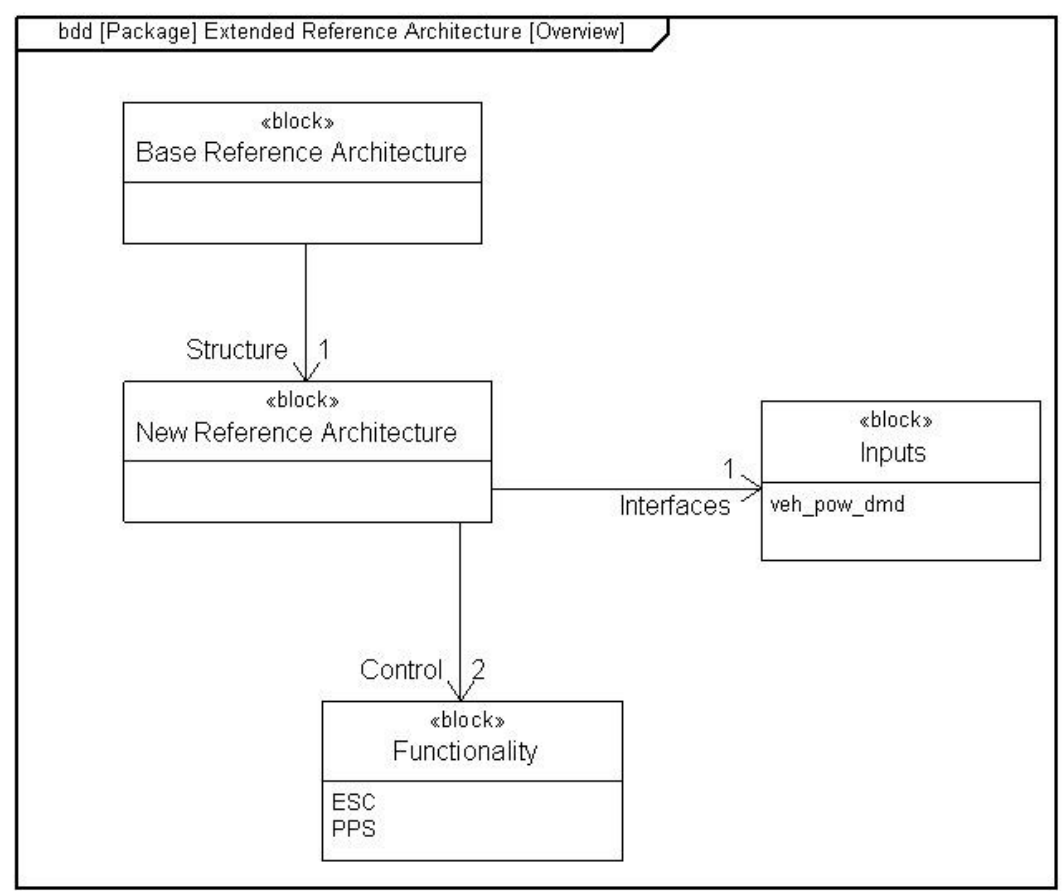

Figure 6: Modifications to the base reference architecture to support the EM of the fixed-voltage network

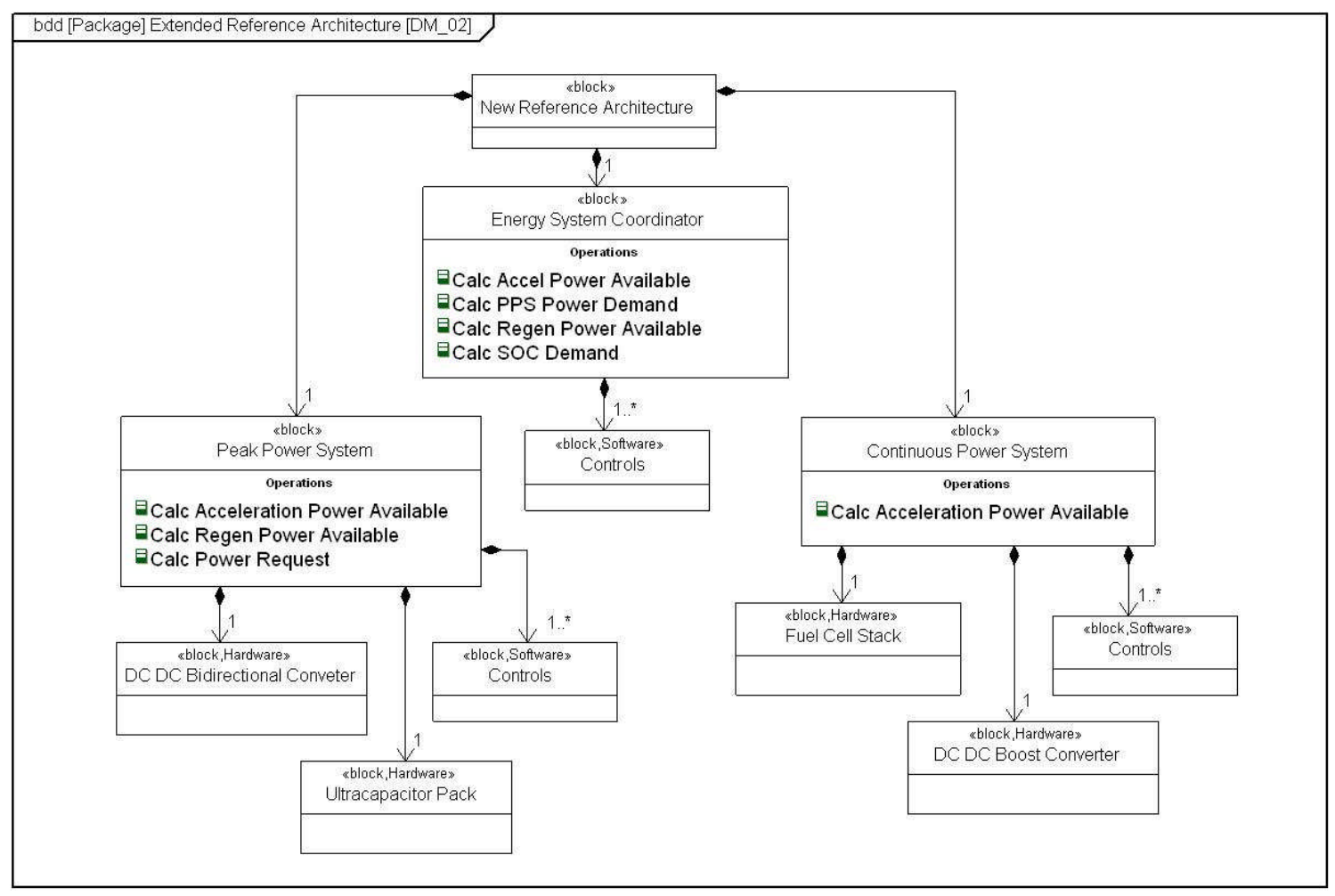

Figure 7: Decomposition model showing the encapsulation of system hardware and controls within the respective reference architecture features 


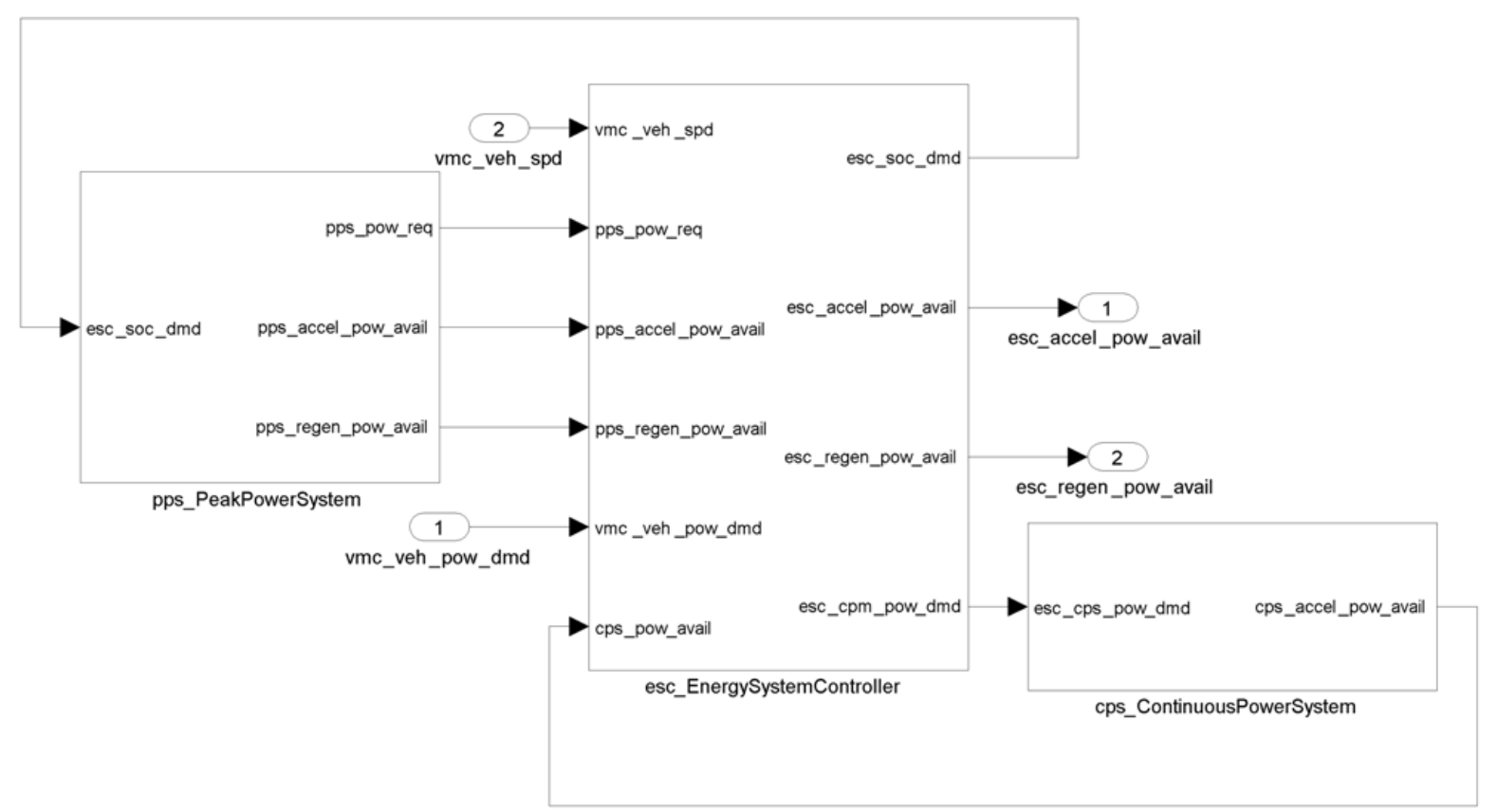

Figure 8: Simulink realisation of the revised reference architecture 

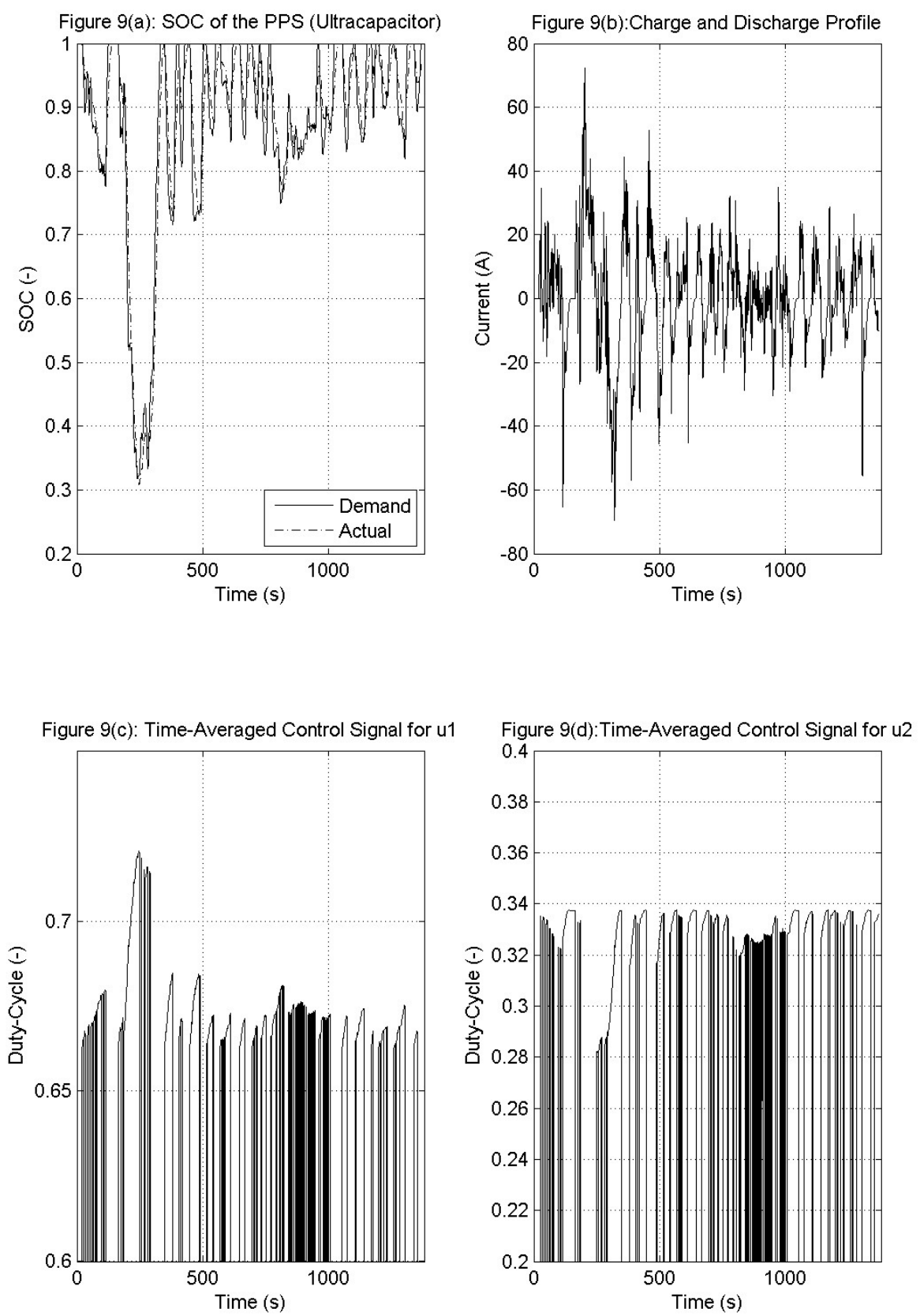

Figure 9: Simulated performance of the revised reference architecture over the UDDS drive cycle. 


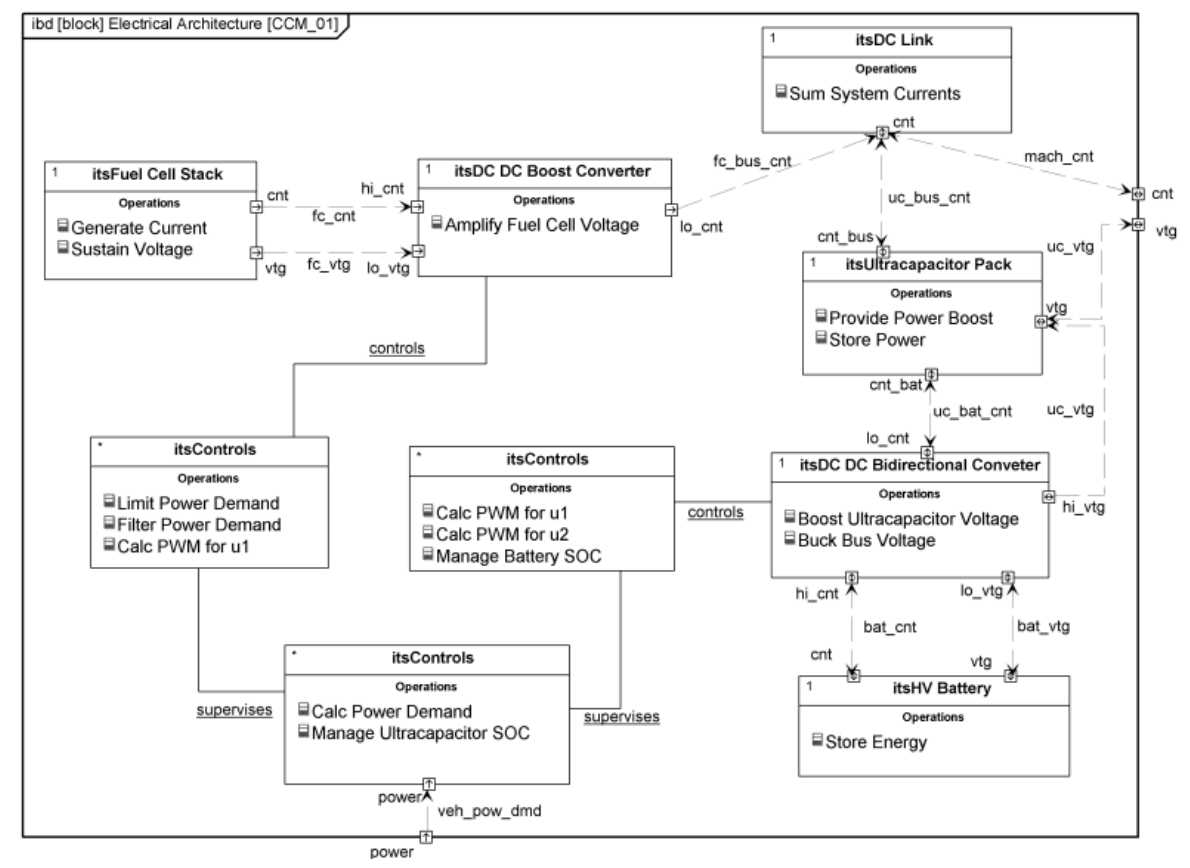

Figure 10: Context and causality model for the revised EV electrical architecture employing a hybrid PPS (HV battery, ultracapacitor and bidirectional converter)

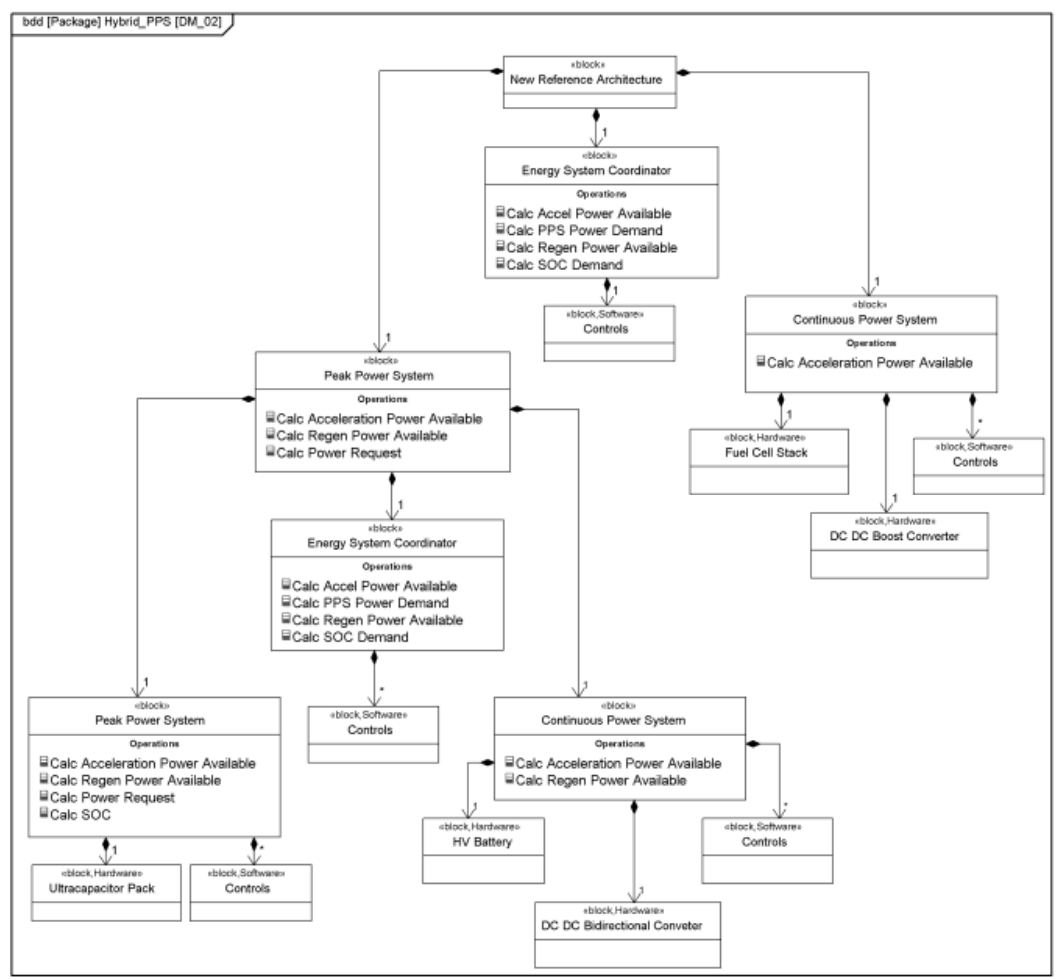

Figure 11: Decomposition model showing the recursion of control features within the reference architecture. 


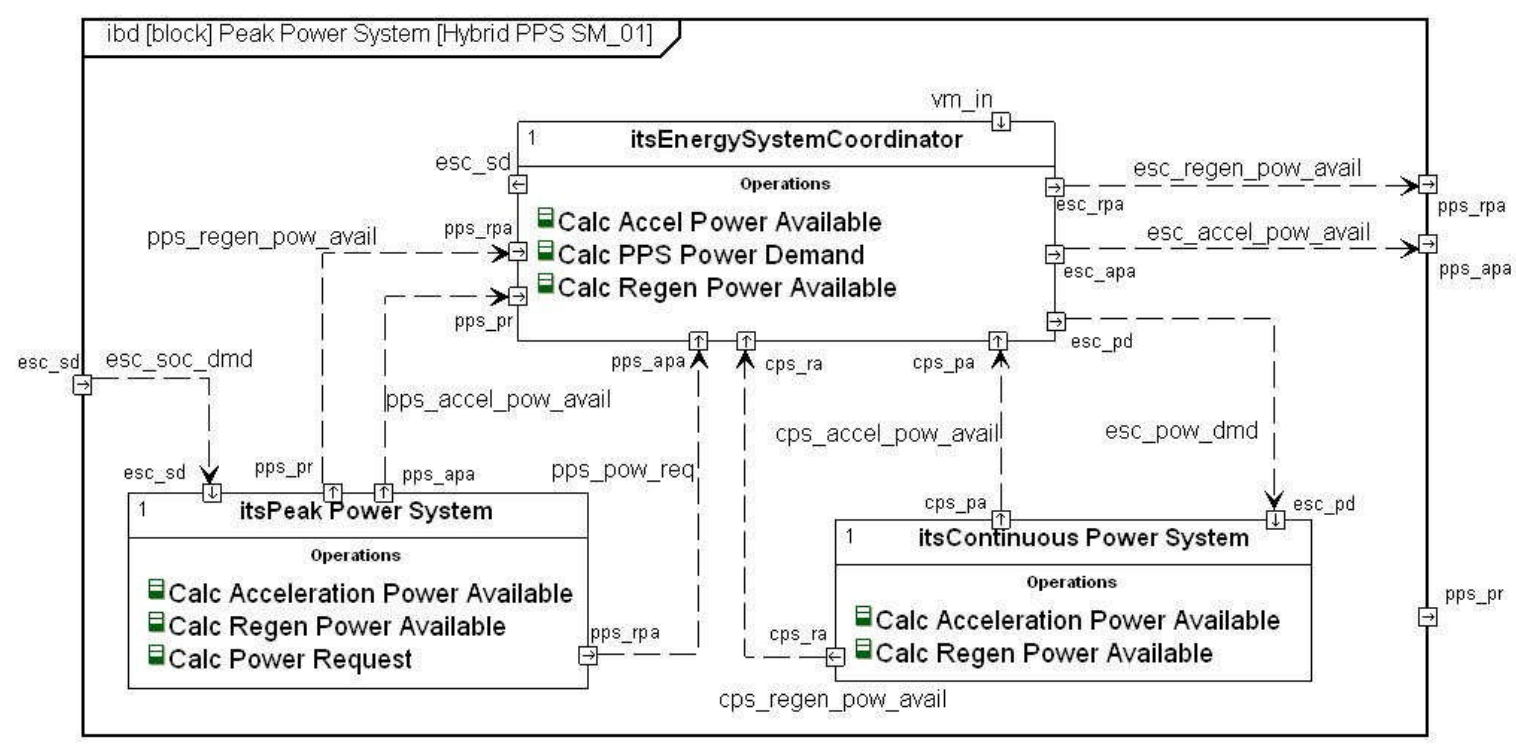

Figure 12: Reference architecture modifications for the hybrid PPS element 

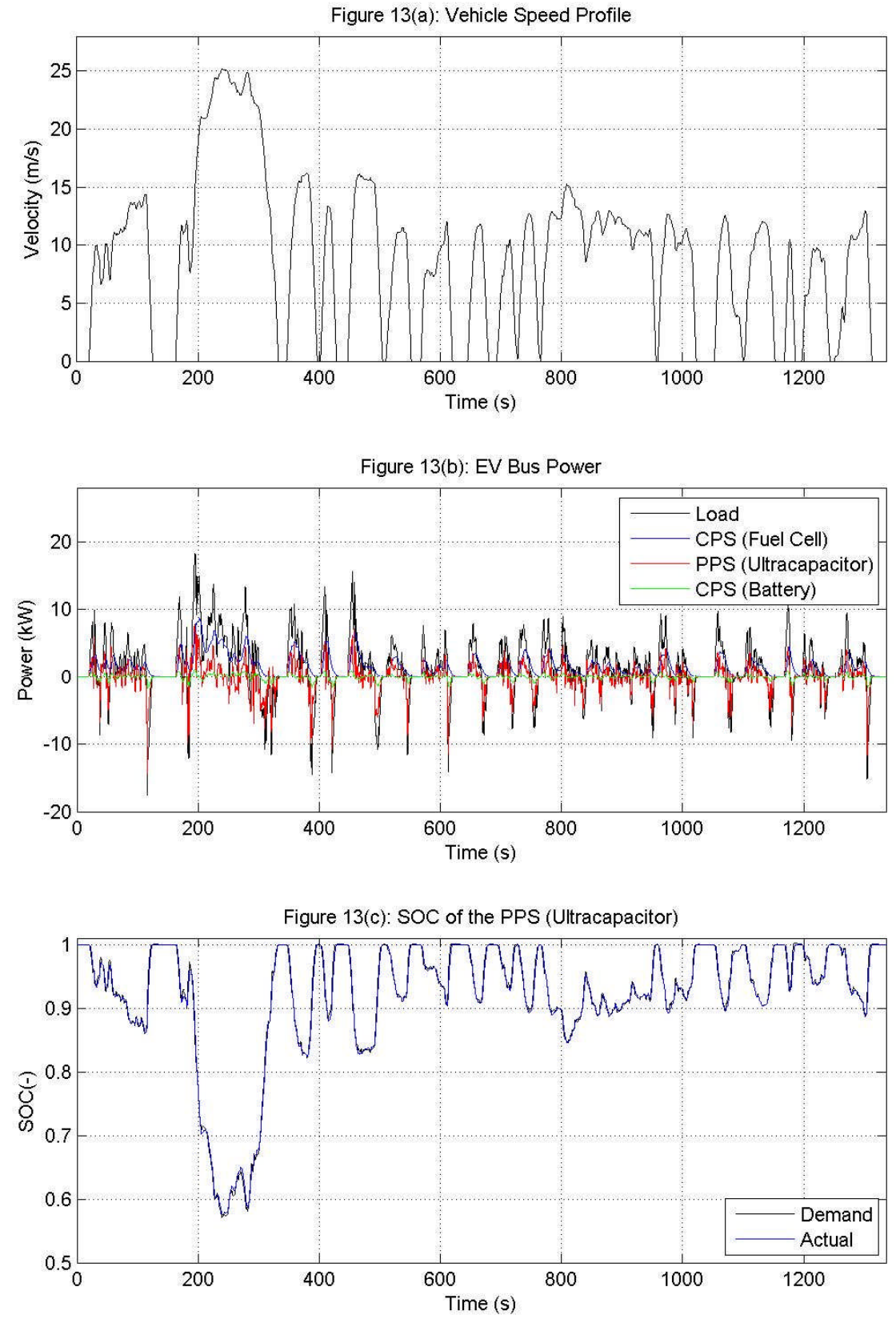

Figure 13: Simulations results of the revised base reference architecture over the UDDS drive cycle 